68 Enrique Pérez-Godoy Díaz

Antonio Javier Moreno-Guerín Baños

Unidad Geriátrica. Centros Sociales Miraflores. Área de acción social. Diputación de Sevilla. Carretera de Miraflores s/n, 41080-Sevilla.

\author{
Correspondencia: \\ Enrique Pérez-Godoy Díaz \\ Av. Emilio Lemos $n^{\circ} 11,2^{\circ} \mathrm{A}$ \\ 41020-Sevilla \\ Tfno.: 954408175,686375739 \\ E-mail: epgodoy@supercable.es
}

\section{Duelo y muerte en cuidadores familiares y profesionales de enfermos de Alzheimer}

\section{RESUMEN}

Como en cualquier proceso crónico y degenerativo abocado a la muerte, la enfermedad de Alzheimer y las demencias provocan en sus cuidadores familiares y profesionales diversos sentimientos, procesos y duelos tanto ante la muerte real como la muerte psíquica.

Utilizando una metodología cualitativa analizamos diversos casos de cuidadores de enfermos de Alzheimer institucionalizados en un centro sociosanitario de donde concluimos que no sólo la muerte real puede provocar un duelo en los cuidadores sino también la pérdida inexorable de capacidades, y en último término su muerte psíquica. Esta muerte afecta también a cuidadores profesionales posiblemente porque les muestra su propia vulnerabilidad como seres humanos, recordándoles su propia muerte y lo que podría percibirse como más cruel: la posibilidad de una muerte en vida.

\section{PALABRAS CLAVE}

Alzheimer, duelo, muerte, cuidador familiar, cuidador profesional.

\section{SUMMARY}

Alzheimer's disease and other dementias can cause in caregivers diverse feelings, processes and mournings, which are lived in different ways. This occurs both in psychological and actual death.

By using a methodology of a qualitative research, we look through some different cases of caregivers whose relatives are living in an institution.

We conclude that not only the actual death can cause a sense of mourning in caregivers of dementia patients, but also the inexorable loss of capacities and eventually their "psychological" death.

This death also possibly affects the professional caregivers because it shows them their vulnerability as human beings. This type of death makes them to think about the possibility of a death in life.

\section{KEY WORDS}

Alzheimer, mourning, death, family caregiver, professional caregiver.

\section{INTRODUCCIÓN}

Como en cualquier proceso crónico y degenerativo abocado a la muerte, la enfermedad de Alzheimer provoca en sus cuidadores distintos sentimientos y procesos psicológicos.

La palabra Alzheimer se asocia frecuentemente a muerte $y$, como consecuencia, la evidencia del grave deterioro de estos pacientes provoca en sus cuidadores familiares un proceso de duelo ante la pérdida de su familiar.

Y es que no sólo la muerte real hace sentir la pérdida, sino también la muerte psíquica consecuencia del grave deterioro y la pérdida de los recuerdos, la desorientación espacial y temporal y la ausencia de reconocimiento de familiares, amigos o incluso de sí mismos, ya que el recuerdo, su historia, es lo que les hace ser lo que son en realidad.

Muestra de ello, es el caso de aquellos enfermos en fase moderada-grave que mediante su deambulación errática, como si de "muertos vivientes" se tratara, van en busca de sus propios recuerdos que no encuentran, o en todo caso, del camino hacia su casa (aunque se encuentren dentro de ella).

Con el siguiente estudio que vamos a presentar pretendemos identificar y analizar algunos de los sentimientos relacionados con la pérdida que experimentan los cuidadores de personas con demencia tanto a lo largo de su proceso demencial como tras la muerte real.

\section{MATERIAL Y MÉTODO}

El presente estudio se ha realizado en familiares de pacientes con algún tipo de demencia (mayoritariamente enfermedad de Alzheimer), en fase avanzada-terminal, con una institucionalización de entre dos meses y tres años y con un importante apoyo familiar. El contexto es una residencia geriátrica, especializada en personas con demencia, antiguo pabellón psicogeriátrico del extinguido Hospital Psiquiátrico de Miraflores. 
Con el objeto de identificar y analizar algunos de los sentimientos relacionados con la pérdida que experimentan los cuidadores, observados por nosotros, de personas con demencia realizamos un estudio observacional descriptivo.

Se ha utilizado una metodología de investigación cualitativa, mediante entrevistas semiestructuradas que fueron elaboradas tras revisión bibliográfica sobre el tema, técnicas de observación participante y sesiones de trabajo del equipo investigador para unificar criterios. En estas entrevistas se plantearon cuestiones sobre la vivencia de la enfermedad previa al ingreso en nuestra residencia, cómo les afectó el diagnóstico de la demencia, qué sintieron ante la muerte de su familiar (en los casos en que habían fallecido) y cómo vivieron los últimos meses de la enfermedad. Estas entrevistas no fueron grabadas por la fuerte implicación emocional de los cuidadores, que no la hubieran permitido o no hubieran profundizado tanto, de forma que fueron transcritas una vez realizadas.

La utilización de estas técnicas permite conocer los espacios donde se desarrolla la relación de los participantes, los patrones de interacción entre los familiares y el personal sociosanitario en general, y enfermería en particular, la descripción de las conductas y los discursos de los participantes y la identificación de los factores que influyen en los distintos tipos de relación.

Basándonos en el modelo de proceso de elaboración del duelo de Elisabeth Kübler-Ross, de esta información escogemos los casos más representativos por ser diferenciales, dentro del corto período de estudio, que fue de unos dos años, así como las frases más ilustrativas utilizadas por los cuidadores para pasar a describir los distintos procesos vivenciales. De esta forma analizamos la vivencia y el contexto familiar y/o relacional.

Este análisis sacará a la luz una diversidad de sentimientos muchas veces contradictorios o paradójicos, con encuentros y desencuentros con la muerte y, por tanto, con la pérdida de esos seres queridos, ya sea real o percibida.

Esta información recogida es fruto del hecho de que el enlace familia-equipo multidisciplinar se establece, frecuentemente, a través del enfermero, que dispone de la valiosa oportunidad de captar el clima de los contactos del paciente y sus familiares durante las visitas (1) y de establecer con ellos una relación de confianza.

\section{RESULTADOS}

\section{Paciente 1}

"Es una viuda cuyo difunto marido aun está vivo"

Con esta frase tan elocuente describió un enfermero en una sesión clínica previa al ingreso en nuestra Unidad a M1, mujer de Paciente 1 de 58 años y enfermo de Alzheimer desde los 52, actualmente en fase moderada-grave e institucionalizado por la imposibilidad de atenderlo continuamente y la dificultad de convivir con la hija de 11 años que comparten debido a los trastornos de conducta que presenta y los graves problemas psicológicos que esta niña está sufriendo.

"Yo ahora me puedo duchar"

Así de gráficamente nos mostró M1 el cambio en su calidad de vida desde que su marido fue institucionalizado. Algo tan simple muestra el continuo estrés y necesidad de control permanente de su marido, de sus necesidades y de las posibles consecuencias de sus trastornos de conducta. Aunque los días laborables él acudía a un centro de día por las mañanas, el resto de días y horas que coincidía con el turno de trabajo de M1 (celadora de un hospital), el Paciente 1 tenía que ser cuidado por su suegra de 80 años.

\section{"Esa es tu vida. Prefiero verlo muerto que así día a día"} Con estas palabras se dirige M2, hija de M1 y del Paciente 1 a su madre cuando ésta le pide que vaya con ella a visitar a su padre. Padre al que casi no ha conocido sano (su enfermedad comenzó cuando ella tenía sólo 6 años) y que con esta frase tan impresionante muestra como ya da por muerto a su padre, reduciendo al mínimo su responsabilidad e implicación hacia él. No en vano seguro que en más de una ocasión habrá tenido que ejercer de madre de su propio padre, aún teniendo ella menos de diez años.

\section{Paciente 2}

"Es lo mejor que le podía haber pasado"

Así expresaba R1 en pleno momento de dolor tras la muerte de su madre, Paciente 2, enferma de Alzheimer en fase severa de 78 años, que tan sólo llevaba dos meses en la unidad, pero que había sufrido un grave deterioro. Esta mujer la visitaba cuantas veces podía y se la llevó a su casa todos los fines de semana que pudo.

\section{"Lo sabía"}

A veces, cuidadoras como R1 que ven la continuidad del proceso degenerativo, vislumbran cuando existe la posibilidad de un desenlace fatal ante pequeños cambios que son a veces muy poco perceptibles, pero que para ellas son desenmascaradores de un proceso que toca a su fin.

"Pero, ¿cómo es posible?, ¿de qué se ha muerto mi madre?" Así entró en la unidad R2, hijo de Paciente 2 y hermano de R1, gritando, llorando y buscando culpables de la pérdida de su madre. Como cuidador secundario no había vivido tan profundamente el proceso de su enfermedad, quizá ni siquiera sabía del "bajón" que había tenido en los últimos días. Por ello, el duelo que estaba viviendo era mucho más intenso que el de su hermana y quizá solo recordaba a su madre en fases previas en las que, aunque enferma, se encontraba mucho mejor y aún se relacionaba con él.

\section{Paciente 3}

$$
\text { "M..., ¿tú me quieres?" }
$$

Esas son las primeras palabras que M1, esposo de Paciente 3 le dirige a su mujer cuando viene a visitarla durante 
70 todo un día. La Paciente 3 padece Alzheimer y se encuentra en fase moderada-severa. Aunque todavía es capaz de contestar con monosílabos es muy posible que le conteste a su marido que sí, sin haber entendido la pregunta. No importa, su marido se da por satisfecho. Además, cuando iba a los grupos de familia le decían que era bueno orientarla y repetirle mucho las cosas.

Aunque M1 tiene más de 80 años, físicamente se encuentra muy bien y entra en la unidad cuando abrimos las puertas y prácticamente todos los días tenemos que pedirle que se vaya. M1 precisa tomar varios autobuses tanto para venir como para volver.

M1 vive en un continuo y permanente duelo. Cada cambio o retroceso en las capacidades de su mujer hace que él viva esa pérdida: negando la evidencia primero, buscando razones o culpables después, apenándose a continuación y aceptando el cambio en ultimo término hasta que otro nuevo cambio vuelva a reactivar el proceso.

"Tenéis un 10"

Le pasó con la dentadura. A pesar de que todo el personal le desaconsejó por su estado de deterioro y los riesgos que podría conllevar el facilitarle una dentadura postiza a su mujer, él insistía en que tenía que hacer todo lo posible. Se les envió a la consulta de un estomatólogo, donde además de revisarle y retirarle alguna pieza se insistió en mostrarle lo innecesario y lo arriesgado del caso. No contento, contactó, a través de su hijo con un catedrático de la facultad de odontología, el cual le insistió en lo mismo. “Tenéis un 10", nos dijo. "El catedrático me ha dicho lo mismo que ustedes”. Lo mismo ocurrió cuando comenzó con los trastornos de la marcha, con los trastornos del tránsito intestinal, etc.

\section{Paciente 4 \\ "CCuales fueron sus últimas palabras?"}

Aparte de los sollozos y agradecimientos por el tiempo dedicado a su padre, F1 tenía interés en conocer las últimas palabras que él había pronunciado. El Paciente 4 era un enfermo de Alzheimer en fase moderada-severa que desde su ingreso mostró trastornos de conducta. Colaboraba poco en su cuidado y debía estar en continua vigilancia pues continuamente trasladaba muebles de sitio y arrancó algún marco de puerta.

F1, su hijo, venía todas las tardes y salía de paseo con su padre, que había vivido mucho tiempo en el campo, ya que conocía su necesidad de salir a tomar el aire. F1 llevaba un diario donde anotaba todos los días las pocas cosas coherentes que su padre decía y que él podía interpretar a través de su propia historia... Tenía interés F1 en anotar en ese diario sus últimas palabras.

\section{Paciente 5}

El Paciente 5 era un residente que llevaba más de 50 años institucionalizado. No estaba claro su diagnóstico principal. Algunos lo diagnosticaron de deficiencia mental, otros de psicosis alcohólica. En su carné de identidad de entonces (años 50) aparecía, con la crueldad la de la época, "Profesión: Anormal". Es muy posible que fuera un pobre deficiente al que más de uno invitara a un vasillo de vino. Terminó viviendo en su manicomio en el que tras reformas y contrarreformas, que él nunca entendió, murió. Su familia pidió que sus cenizas fueran esparcidas en uno de los patios de la unidad, donde él siempre había vivido.

"Pero ¿qué pasa aquí?, todo el mundo llorando!"

Estas fueron las palabras de un director que no entendía que todo el personal trabajara intentando disimular las lágrimas. También los profesionales sienten la pérdida y tienen a veces la necesidad de desahogarse, cuando al pasar por su habitación ya no le escuchan sus demandas de atención. Los profesionales no somos de piedra, y no podemos asistir indemnes a los cambios y sucesos que pasan por delante de nosotros.

\section{Nuevas demandas}

"Q Qué enfermedad más cruel!

Frase que se escucha con mucha frecuencia en nuestra unidad, referida a la enfermedad de Alzheimer. Curiosamente, sólo se escucha desde que llevamos varios ingresos de pacientes con edades inferiores a 60 años y en un estado muy avanzado de deterioro. Alguno, incluso con hijos pequeños.

También, quienes más repiten esta queja, son profesionales de mediana edad o incluso mayores que los propios enfermos, lo que puede hacer que se identifiquen más con estos pacientes y con sus familias. ¿Es sólo pena lo que sienten por ellos? ¿No será que se evidencia la vulnerabilidad del ser humano de padecer esta u otras enfermedades donde se pierde el contacto con la realidad y se puede entrar en una muerte psíquica?

\section{DISCUSIÓN Y CONCLUSIONES}

De los casos analizados podemos concluir que no sólo la muerte real puede provocar un duelo en los cuidadores de pacientes con demencia sino también la pérdida inexorable de capacidades y en último término su muerte psíquica, aunque las limitaciones metodológicas de este estudio impiden generalizar y hacer transferencia de estas conclusiones.

Esta muerte afecta también a los cuidadores profesionales, no tanto por la relación con el enfermo, sino porque le muestra su propia vulnerabilidad como ser humano, recordándole su propia muerte y lo que podría percibirse como más cruel: la posibilidad de una "muerte en vida”.

Estas conclusiones nos hacen reflexionar sobre las distintas formas y grados de intensidad de vivenciar la pérdida de un ser querido que padece demencia y quizá se podría profundizar más analizando casos de familias cuyo miembro con demencia vive en su propio domicilio. 


\section{BIBLIOGRAFÍA}

1. Crespo Benítez J et al. La relación interpersonal. Relaciones del enfermero con el paciente, la familia y el equipo terapéutico. En: Bobes J et al. Enfermería Psiquiátrica. Madrid: Doyma; 2000.

2. Schulz R, Belle S et al. Long-term care placement of dementia patients and caregiver health and well-being. JAMA 2004; 292: 961-7.

3. Carnós L et al. Ansiedad en los cuidadores principales de personas mayores. Rev Enferm Clín 2005; 5: 251-6.

4. Boada Rovira M, Tárraga Mestre L. Alzheimer. La memoria está en los besos. Barcelona: Ed. Mayo; 2002.
5. Flórez Lozano JA. El médico ante la familia y el enfermo de Alzheimer. 71 Madrid: Idepsa; 1999.

6. Boada M, Tárraga L. Cuadernos de repaso. Ejercicios prácticos de estimulación cognitiva para enfermos de Alzheimer en fase moderada. Barcelona: Ed. Glosa; 2003

7. Bayés R. Psicología del sufrimiento y de la muerte. Barcelona: Martínez Roca; 2001.

8. Kübler-Ross E. Sobre la muerte y los moribundos. Barcelona: Random House-Mondadori; 2005. 